\title{
The Herb-Drug Interaction of Clopidogrel and Xuesaitong Dispersible Tablet by Modulation of the Pharmacodynamics and Liver Carboxylesterase 1A Metabolism
}

\author{
Shitang Ma $\left(\mathbb{D},{ }^{1,2}\right.$ Guoliang Dai, ${ }^{3}$ Xiaolin Bi, ${ }^{3}$ Meirong Gong, ${ }^{3}$ Chenggui Miao, ${ }^{1}$ Hao Chen, \\ Liangliang Gao, ${ }^{1}$ Weiman Zhao, ${ }^{4}$ Tianjun Liu $\mathbb{D}^{2},{ }^{2}$ and Ningru Zhang $\mathbb{D}^{4}$ \\ ${ }^{1}$ Life and Health College, Anhui Science and Technology University, China \\ ${ }^{2}$ Institute of Bio-Medical Engineering, Chinese Academy of Medical Sciences, China \\ ${ }^{3}$ Nanjing University of Chinese Medicine, China \\ ${ }^{4}$ The First Affiliated Hospital of Bengbu Medical College, China \\ Correspondence should be addressed to Tianjun Liu; liutianjun@hotmail.com and Ningru Zhang; yiaocaoyaya@163.com
}

Received 28 June 2018; Revised 31 August 2018; Accepted 1 October 2018; Published 18 October 2018

Academic Editor: Ling Yang

Copyright ( 2018 Shitang Ma et al. This is an open access article distributed under the Creative Commons Attribution License, which permits unrestricted use, distribution, and reproduction in any medium, provided the original work is properly cited.

\begin{abstract}
Objective. Clopidogrel and Xuesaitong dispersible tablet (XST) have been clinically proven to be effective for treating cardiocerebrovascular disease. The present study was to investigate the herb-drug interaction of Clopidogrel and XST by modulation of the pharmacodynamics and liver Carboxylesterase 1A(CES1A) metabolism. Methods. 30 male SD rats were randomly divided into a control group (equal volumes of saline, 6 rats for mRNA analysis), a clopidogrel group (clopidogrel with dose $30 \mathrm{mg} / \mathrm{kg}$ ), and a combination group (clopidogrel and XST, with dose 30 and $50 \mathrm{mg} / \mathrm{kg}$ respectively, each group continuous administration once daily for 30 days). The clopidogrel and combination group comprised 12 rats, with 6 designated for mRNA analysis and 6 for the pharmacokinetic study. The 2-bromo-3'-methoxyacetophenone- (MPB-) derivatized clopidogrel active thiol metabolite (CAMD) was measured by UHPLC-MS/MS for pharmacokinetics $(n=6)$. The expression of CES1A mRNA was examined with real-time RT-PCR $(n=6)$. Molecular simulation was used to investigate the inhibition effect of XST on the CES1A protein. The CAMD pharmacodynamics and CES1A metabolism were investigated to evaluated the herb-drug interaction. Results. Clopidogrel and XST coadministration appreciably increased the Cmax, AUC, and MRT of CAMD. However, the expression of CES1A mRNA was decreased accordingly. It also indicated that the bioactive components in XST had good interaction with the CES1A metabolism target by molecular simulation. The animal study indicated that clopidogrel and XST coadministration produced significant herb-drug interactions at active CAMD pharmacokinetic and CES1A metabolic enzyme aspect. Conclusion. 30-days dose of coadministration altered hepatic CES1A protein and resulted in reduced plasma levels of active CAMD. both the decreased CES1A mRNA expression and the inhibition on the protein were due to the combination of XST, which accordingly upregulated the pharmacokinetics of plasma active CAMD.
\end{abstract}

\section{Introduction}

Clopidogrel, a second generation thienopyridine P2Y12 inhibitor, has been the standard-of-care for percutaneous coronary intervention (PCI) and/or acute coronary syndrome (ACS) [1-3]. Although the drug is generally considered safe and effective, many clinical studies have shown that approximately $5-40 \%$ of patients displayed inadequate antiplatelet responses $[2,4]$. This result has increased platelet reactivity and cardiovascular events during treatment [5]. These patients need to seek alternative antiplatelet therapies $[3,6,7]$, e.g., Chinese medicine combinations.

Clopidogrel is an inactive prodrug that requires enzymatic conversion by a number of carboxylesterases (CESs) enzymes and cytochrome P450 (CYP) enzymes [8-10]. During clopidogrel metabolism [11, 12], carboxylesterase 1A (CES1A) begins by hydrolyzing approximately $85-90 \%$ of the prodrug to an inactive carboxylic acid metabolite [13]. A 


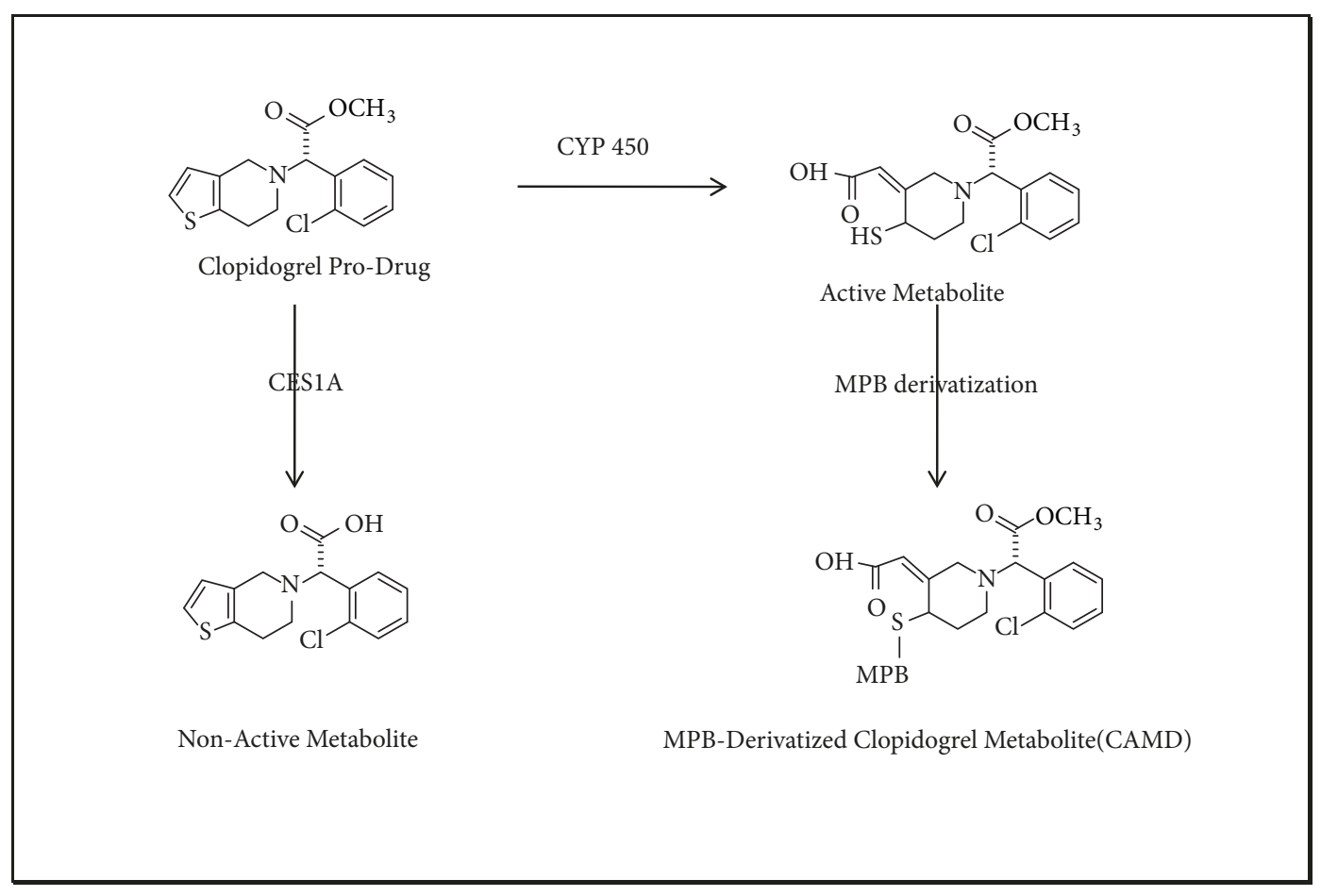

FIGURE 1: Formation of the active CAMD metabolite.

portion of the prodrug is transformed into the inactive 2-oxoclopidogrel [6], and the remainder is oxidized to the active thiol metabolite by CYP enzymes (Figure 1) [14, 15]. Only approximately $2 \%$ of the clopidogrel dose reaches systemic circulation where it becomes available through irreversible binding to the platelet $\mathrm{P} 2 \mathrm{Y} 12$ receptor to have an antiplatelet aggregation effect [16].

Our previous study found that multiple dose (30 days) of clopidogrel altered hepatic CES1A in rats, which resulted in elevating the serum inactive carboxylic metabolite [17]. When a Chinese medicine recipe containing Salvia Miltiorrhiza, Radix Notoginseng, and Borneol combined with FDDP, the activity of CES1A was partially inhibited based on molecular simulation experiments [18], which have the pharmacokinetic result of decreasing levels of the inactive carboxylic acid metabolite [19].

Xuesaitong (XST, Chinese drug Z20050467), extracted from Panax notoginseng (Burk.) F.H. Chen (Sanqi), was widely used in TCM hospitals [20, 21]. Ginsenoside Rg1, Rd, and notoginsenoside $\mathrm{R} 1$ were its main active components [22, 23]. XST was used to treat cerebral infarction and ischemia, coronary heart disease, and atherosclerosis [24, 25]. However, there have not been any publications describing a herbaldrug effect of clopidogrel with XST through modulation of target metabolism and pharmacokinetics. The present study investigated the rationale of combined applications and the drug-herb effects on target metabolism (by RT-PCR for the CES1A mRNA expression and molecular simulation for the metabolic enzyme) and pharmacokinetics of CAMD(by UHPLC-MS/MS).

\section{Experimental}

\subsection{Materials and Methods}

2.1.1. Reagents and Materials. The 2-bromo-3'-methoxyacetophenone- (MPB-) derivatized clopidogrel thiol metabolite (CAMD, lot No. 5-MNZ-195-23), MPB (lot No. 151910100), and internal standard (IS, guanosine, lot No. 111977-201501) were purchased from the Toronto Research Chemicals, Sigma, and the Chinese National Institute for the Control of Pharmaceutical and Biological Products (NICPBP), respectively. XST tablets (0.5 g/tablet, lot No. DHB1606, expiring before May 2018), containing $13.6 \mathrm{mg} / \mathrm{g}$ ginsenoside Rg1, $8.6 \mathrm{mg} / \mathrm{g}$ ginsenoside $\mathrm{Rd}$, and $7.8 \mathrm{mg} / \mathrm{g}$ ginsenoside R1, were manufactured by the Yunnan Dali Medicine Factory (Yunnan, China). Clopidogrel tablets (25 mg/tablet, lot No. AA20150207, expiring before Jan 2018) were manufactured by Shenzhen Salubris Pharmaceuticals Co. (Shenzhen, China). Acetonitrile and methanol were HPLC grade (Merck, USA). The ultrapure water used for UHPLC-MS/MS was from a Milli-Q water purification system (Millipore, USA).

The RNAiso Plus reagent, PrimeScript ${ }^{\mathrm{TM}}$ II 1st Strand cDNA Synthesis Kit, SYBR Premix Ex Taq ${ }^{\mathrm{TM}}$ II kit and primer were provided by the TaKaRa Biotechnology Company (Takara, Japan).

2.2. Animal Treatment. Thirty male SD rats, body weights 220-300 g (License No. SCXK, Jiangsu Province, China, 20140007) were purchased from Suzhou Industrial Park Aier Matt Technology Co. Ltd. (Suzhou, China). All rats were pathogenfree and acclimated for at least one week. The rats were housed 
in an environmentally controlled room with a temperature of $20 \pm 2^{\circ} \mathrm{C}$, light from $06: 30 \mathrm{~h}$ to $18: 30 \mathrm{~h}$, and humidity of 60 $\pm 5 \%$. All rats were fed standard rodent chow and water ad libitum. This procedure was approved by the Animal Ethics Committee of the Nanjing University of Chinese Medicine.

Three groups were randomly assigned according to a parallel study. The control group consisted of 6 rats (oral administration of equal volumes of saline) for mRNA analysis. The clopidogrel group received $30 \mathrm{mg} / \mathrm{kg}$ of clopidogrel orally that comprised 12 rats, with 6 designated for mRNA analysis and 6 for the pharmacokinetic study. The combination group had 12 rats, with 6 allocated for mRNA analysis and 6 for the pharmacokinetics, and all orally received both clopidogrel at $30 \mathrm{mg} / \mathrm{kg}$ and XST at $50 \mathrm{mg} / \mathrm{kg}$. The rats in each group were treated for 30 days. The rats fasted for $12 \mathrm{~h}$ before the experiment but had unlimited access to water. The drugs were suspended in saline before oral administration to rat.

2.3. Experimental Procedure. All rats were continuously intragastrically fed each drug for 1 month, as described in "animal treatment". For the pharmacokinetic analysis, 6 rats each were selected from the clopidogrel and combination group. Blood plasma samples of approximately $150 \mu \mathrm{L}$ were collected in $500 \mathrm{mM}$ of MPB pretreated EDTA centrifuge tubes from the fossa orbitalis vein at $0,0.083,0.25,0.5,0.75,1$, $2,3,4,6,8,12$, and $24 \mathrm{~h}$ before and after drug administration on the thirty-first day. Then, the samples were centrifuged for $10 \mathrm{~min}$ at $4000 \mathrm{rpm}$, and the supernatant was transferred to labeled plastic vials and stored at $-20^{\circ} \mathrm{C}$ until analyzed.

The remaining 18 rats from the three groups were used for mRNA analysis. After overnight fasting, the rats were sacrificed under anesthesia by i.p. administration of a 0.4 $\mathrm{mL} / 100 \mathrm{~g}$ dose of a $10 \%$ chloral hydrate solution. The samples were finally collected as follows. The rat livers were removed and weighed. Portions of the liver samples were stored at $-80^{\circ} \mathrm{C}$ for further mRNA biochemical assays [26].

2.4. The Stock Solutions and Plasma Sample Preparation. Master stock solutions were prepared by individually dissolving CAMD and IS in methanol at free-base equivalent concentrations of $1000 \mu \mathrm{g} / \mathrm{mL}$. Working solutions were prepared from the stock solutions by dilution in methanol. All working solutions were stored at $4^{\circ} \mathrm{C}$. For each run, calibration standards in drug-free rat EDTA plasma were freshly prepared in duplicate at concentrations of $66,41.96$, $10.24,5.12,2.56,1.28$, and $0.64 \mathrm{ng} / \mathrm{mL}$ for CAMD. QC samples were prepared at $49.5,30,1.8 \mathrm{ng} / \mathrm{mL}$. All the standard calibration samples and QC samples were stored at $-20^{\circ} \mathrm{C}$.

All frozen standards and samples were thawed on wet ice before homogenization. A $50 \mu \mathrm{L}$ aliquot of each plasma sample and $10 \mu \mathrm{L}$ IS solution $(860.00 \mathrm{ng} / \mathrm{mL})$ were transferred into a $1.5 \mathrm{~mL}$ centrifuge tube where protein precipitation was performed by adding $200 \mu \mathrm{L}$ of methanol. The mixture was vortexed for $5 \mathrm{~min}$ and then centrifuged at $12000 \mathrm{rpm}$ for 10 min before the supernatant was transferred to the UHPLCMS/MS for analysis [27, 28].

2.5. UHPLC-MS/MS Instrumentation and Conditions. Chromatographic separations were performed with an Agilent
HPLC 1290 system (Agilent, USA) consisting of a quaternary pump, an online degasser, and an autosampler. The chromatographic separation was performed on a Phenomenex Gemini C18 reversed phase analytical column (110 $\AA, 3 \mu \mathrm{m}$ particle size, $2.0 \mathrm{~mm}$ I.D. $\times 100 \mathrm{~mm}$ ). The mobile phase consisted of methanol and an aqueous solution of $0.1 \%$ formic acid $(80: 20 ; v / v)$ at a flow rate of $0.2 \mathrm{~mL} / \mathrm{min}$. The autosampler temperature was maintained at $4^{\circ} \mathrm{C}$, and the injection volume was $5 \mu \mathrm{L}$. The total $\mathrm{LC}$ run time was $5 \mathrm{~min}$, with a column temperature of $30^{\circ} \mathrm{C}$.

Detection of the analytes and IS was performed on a G6430 tandem quadrupole mass spectrometer (Agilent, USA) with an electrospray ionization (ESI) interface in positive ion mode. Multiple reaction monitoring (MRM) was used to monitor precursor to product ion transitions of $m / z 504.1 \longrightarrow 211.7$ for CAMD and $m / z 284.1 \longrightarrow 152.1$ for IS. the source parameters were a capillary voltage of $3.5 \mathrm{kV}$, a gas temperature of $350^{\circ} \mathrm{C}$, and a gas flow of $10 \mathrm{~L} / \mathrm{min}$. The compound dependent parameters of fragmentor and collision energy were optimized at $130 \mathrm{~V}$ and $10 \mathrm{~V}$ for CAMD and at $90 \mathrm{~V}$ and $10 \mathrm{~V}$ for the IS, respectively. Dwell time set was $200 \mathrm{~ms}$ for CAMD and the IS. The resultant data were processed using MassHunter software (version B.05.00, Agilent).

2.6. UHPLC-MS/MS Method Validation. The method was validated in terms of linearity, accuracy and precision, selectivity, matrix effect (ME), recovery, and stability according to the guidelines for bioanalytical method development recommended by the US Food and Drug Administration and related literature for CAMD detection $[14,29]$.

2.7. Pharmacokinetic Analysis. The blood samples in the pharmacokinetic analysis were prepared, and the CAMD concentrations were assessed by the validated LC-MS/MS method. Pharmacokinetic parameters were calculated using the Drug and Statistic (DAS) 3.0 pharmacokinetic software (Chinese Pharmacological Association, Anhui, China).

2.8. RNA Extraction and Real-Time RT-PCR. Total RNA was extracted from $100 \mathrm{mg}$ of the livers using the RNAiso Plus reagent (Takara, Japan) $[13,30]$. Then, $0.5 \mu \mathrm{g}$ of the extracted RNA was reverse transcribed into first-strain complementary DNA (cDNA) using a PrimeScript ${ }^{\mathrm{TM}}$ II 1st Strand cDNA Synthesis Kit (Takara, Japan). Real-time RT-PCR was then performed using SYBR Premix Ex Taq ${ }^{\mathrm{TM}}$ II kit (Takara, Japan) on the Mx3000pTM Real-time RT-PCR system (Stratagene, Mx3000p, USA) following the $2^{-\Delta \Delta C t}$ method. The following primers were used for the analysis of the rat samples: CES1A:5'-CTACCCACCTATGTGCTCCC-3'(sense) and 5'-GCCCAGGCGATACTGAATGAC-3'(antisense); $\beta$ ACTIN:5'- CACTATCGGCAATGAGCG -3'(sense) and 5'AGGAGCCAGGGCAGTAATC - $3^{\prime}$ (antisense). The relative expression of the genes was normalized using B-ACTIN as the internal reference.

2.9. Statistical Analysis. Each value obtained from experiments was expressed as the mean $\pm \mathrm{SE}, \mathrm{n}=6$. The mean comparisons for each group from the pharmacokinetic and 


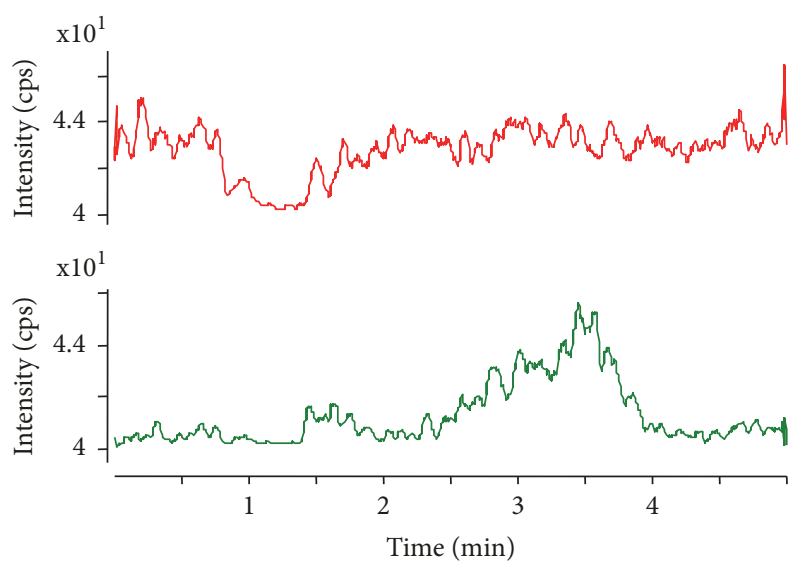

(a)

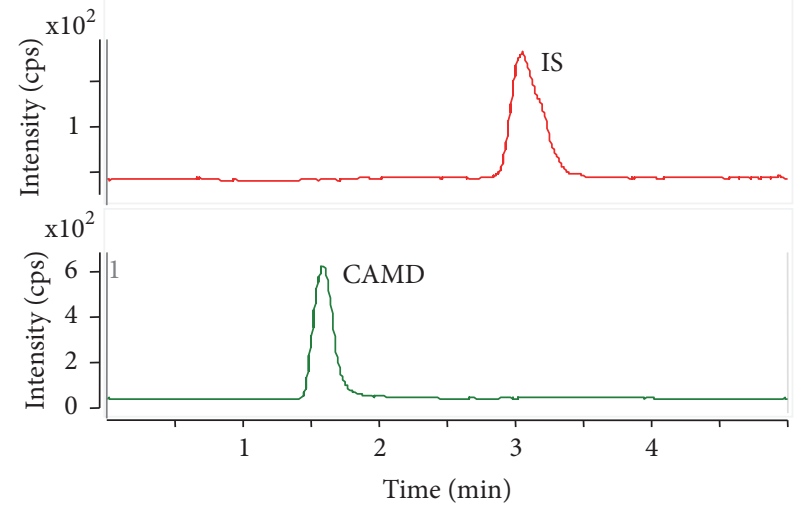

(b)

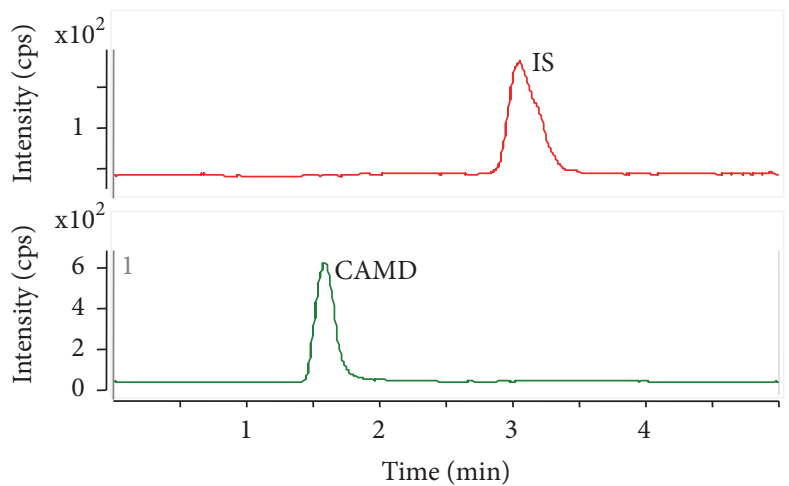

(c)

FIGURE 2: Chromatograms of CAMD and IS. (a) A blank rat plasma sample; (b) a blank plasma spiked with CAMD and IS at the LLOQ; (c) a rat plasma sample after an oral administration of XST $(50 \mathrm{mg} / \mathrm{kg})$ and clopidogrel $(30 \mathrm{mg} / \mathrm{kg})$ at intervals of $0.5 \mathrm{~h}$.

mRNA Expression were performed using Student's $t$-test and one-way ANOVA respectively. Differences with $P<0.05$ were considered statistically significant.

2.10. Molecular Simulation. Schrodinger Maestro 8.5 was used to investigate the molecular simulation. The XST bioactive components (ginsenoside Rg1, Rd and notoginsenoside R1) and the CES1A protein (PDB ID 1MX1) were prepared with LigPrep and protein preparation wizard, respectively. Then, the above materials were subjected to Glide based three-tiered in silico target screening strategy by two stages of the docking protocol, High Throughput Virtual Screening (HTVS), and Standard Precision (SP).

\section{Results and Discussion}

3.1. Pharmacokinetic Method Validation. The protein precipitation sample preparation in combination with UHPLCMS/MS detection provided good selectivity for the CAMD analytes and the IS. Figure 2 shows the typical chromatograms of a blank plasma sample, a blank plasma sample spiked with CAMD analyte at the LLOQ and the IS, and the TIC for the combination group plasma after multiple dose for 30 days at $0.5 \mathrm{~h}$ intervals. No significant interfering peaks were observed at the retention times of the analyte or the IS.
The retention times for CAMD and the IS were 1.59 and 3.08 min, respectively.

The assay was validated over the nominal concentration range of $0.64-66.00 \mathrm{ng} / \mathrm{mL}$. The calibration curve correlation coefficients $\left(R^{2}\right)$ were 0.998 8. A typical calibration curve equation was $Y=28.067 X+0.0018$, which indicated a good fit of the calibration data to the regression lines. The lowest concentration at $\mathrm{S} / \mathrm{N}$ ratios of 10 with the $\mathrm{RSD}<20 \%$ was taken as LLOQ and was found to be $0.64 \mathrm{ng} / \mathrm{mL}$ for CAMD (Table 1).

The extraction recoveries of the analytes from plasma at the three QC concentration levels were 90.20\%-93.10\%. The matrix effects at three QC levels were in the range of $92.40 \%$ $101.20 \%$ with RSD values below $6.70 \%$. In this assay, the intraand interday precisions were measured to be below $7.10 \%$ and $6.30 \%$, respectively, with relative errors from $-2.30 \%$ to $5.30 \%$ (Table 2).

The analytes were stable in the plasma samples for at least $6 \mathrm{~h}$ at room temperature or on ice. No significant degradation was observed when extracted plasma samples were kept at $4^{\circ} \mathrm{C}$ in the autosampler for up to $24 \mathrm{~h}$.

3.2. Application of the Validated Assay to the Pharmacokinetic Study. The validated UHPLC-MS/MS assay was successfully applied to the quantitation of CAMD in rat plasma samples. 
TABLE 1: Mean matrix effect and recovery of CAMD in rat plasma $(n=6)$.

\begin{tabular}{|c|c|c|c|c|}
\hline Concentration $(\mathrm{ng} / \mathrm{mL})$ & Matrix effect (\%) & RSD (\%) & Recovery (\%) & RSD (\%) \\
\hline 1.80 & 92.40 & 4.90 & 90.20 & 5.10 \\
\hline 30.00 & 101.20 & 6.70 & 93.10 & 6.30 \\
\hline 49.50 & 98.30 & 5.30 & 92.30 & 2.30 \\
\hline
\end{tabular}

TABLE 2: Precision and accuracy of CAMD assay in rat plasma $(n=6)$.

\begin{tabular}{lcccc}
\hline $\begin{array}{l}\text { Concentration } \\
(\mathrm{ng} / \mathrm{mL})\end{array}$ & $\begin{array}{c}\text { Intra-day } \\
\text { Precision }\end{array}$ & $\begin{array}{c}\text { Inter-day } \\
\text { (\%, RSD) }\end{array}$ & $\begin{array}{c}\text { Precision } \\
(\%, \mathrm{RE})\end{array}$ & $\begin{array}{c}\text { Accuracy } \\
(\%, \mathrm{RE})\end{array}$ \\
\hline 0.64 & 7.10 & 4.30 & 4.80 & 3.30 \\
1.80 & 6.20 & -2.30 & 6.30 & 4.40 \\
30.00 & 5.60 & 5.30 & 2.90 & 3.70 \\
49.50 & 6.90 & 3.80 & 4.90 & 4.90 \\
\hline
\end{tabular}

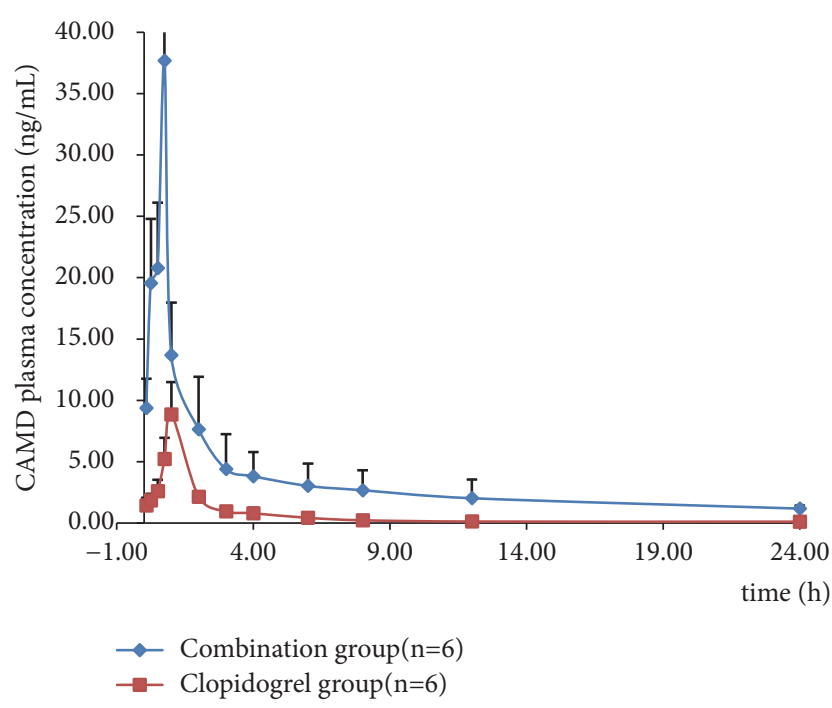

Figure 3: Mean plasma concentration-time profiles of CAMD in rats $(n=6)$ after continuous oral administration of clopidogrel (30 $\mathrm{mg} / \mathrm{kg}$ ) with or without XST (50 mg/kg).

The mean plasma concentration-time profiles are illustrated in Figure 3 and pharmacokinetic parameters are presented in Table 3.

The $\mathrm{AUC}_{(0-24)}$ and $\mathrm{AUC}_{(0-\infty)}$ in rats after combinational administration of clopidogrel and XST were $84.13 \pm 4.72$ and $107.03 \pm 5.31$, which were significantly higher than that in clopidogrel group $(15.91 \pm 2.93$ and $16.31 \pm 3.15, P<0.05)$. The mean peak concentration $\left(\mathrm{C}_{\max }\right)$ was achieved with significantly high value of $37.71 \pm 6.34$ in combinational group, which was detectable in normal rat plasma with low value of $8.92 \pm 2.63$ in clopidogrel group $(P<0.05)$. Similarly, the MRT of the CAMD were also significantly increased $(P<0.05)$. There were no significant changes observed in $\mathrm{T}_{\max }, \mathrm{T}_{1 / 2}$, and $\mathrm{Vd} / \mathrm{F}$ of analyte concentration in the two groups.

In the present investigation, it was found that the pharmacokinetic parameters of CAMD in combinational group were different from those in clopidogrel rats. Clopidogrel and

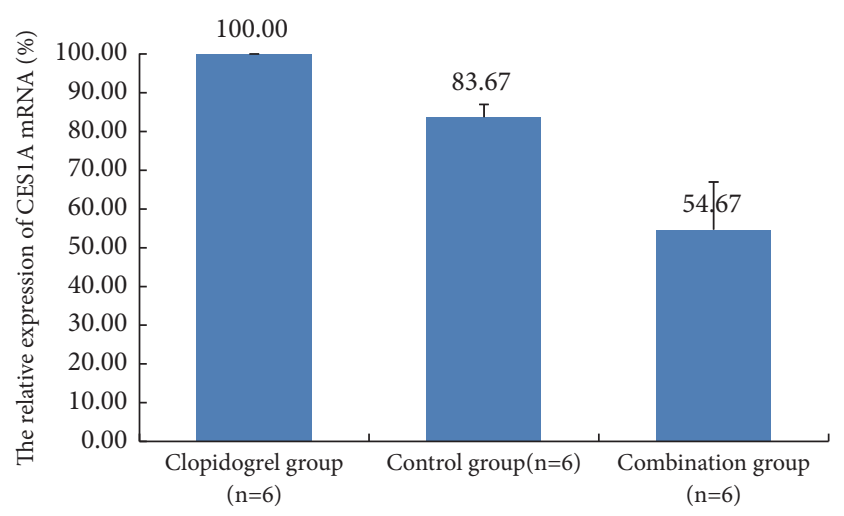

FIgURE 4: The relative expression of CES1A mRNA in rat livers after 30 days of administration in each group, as determined by RT-PCR.

XST coadministration appreciably increased the Cmax, AUC, and MRT of CAMD (the active thiol metabolite). The above results indicated that combination with XST could increase the plasma concentrations of CAMD in rats.

3.3. CES1A mRNA Levels in Liver Tissues of Rats. The expression of the CES1A enzyme mRNA was measured by Real-Time RT-PCR in rat liver to evaluate the impact of the XST on clopidogrel hydrolysis. As shown in Figure 4, compared to the control group, there was a significant acute increase in the relative expression of CES1A mRNA in the clopidogrel group $(83.67 \pm 3.30$ versus 100$)$, which indicated that 30-day dose of clopidogrel would increase the level of the CES1A mRNA in vivo [17]. This would upregulate the serum concentration of inactive clopidogrel carboxylic metabolites and downregulate the serum concentration of active CAMD metabolites correspondingly. Compared to the clopidogrel group, the mRNA expression level was downregulated when combined with XST $(54.67 \pm 12.29$ versus $100, P<0.05)$. As the CES1A enzyme is responsible for clopidogrel hydrolysis, changes in expression of the CES1A mRNA would result in altered plasma concentrations of the clopidogrel metabolite. 
TABLE 3: Pharmacokinetic parameters of CAMD after intragastric administration of clopidogrel alone or coadministration of clopidogrel and XST to rats.

\begin{tabular}{lcc}
\hline Parameter & Clopidogrel group $(n=6)$ & Combination group $(n=6)$ \\
\hline $\mathrm{T}_{\max }(\mathrm{h})$ & $1.00 \pm 0.85$ & $0.75 \pm 0.48$ \\
$\mathrm{C}_{\max }(\mathrm{ng} / \mathrm{mL})$ & $8.92 \pm 2.63$ & $37.71 \pm 6.34 *$ \\
$\mathrm{AUC}_{(0-24)}$ & $15.91 \pm 2.93$ & $84.13 \pm 4.72 *$ \\
$\mathrm{AUC}_{(0-\infty)}$ & $16.31 \pm 3.15$ & $107.03 \pm 5.31 *$ \\
$\mathrm{MRT}_{(0-24)}(\mathrm{h})$ & $3.84 \pm 0.94$ & $6.39 \pm 1.35 *$ \\
$\mathrm{MRT}_{(0-\infty)}(\mathrm{h})$ & $4.43 \pm 1.24$ & $7.29 \pm 1.40 *$ \\
$\mathrm{Vd} / \mathrm{F}(\mathrm{L})$ & $9.53 \pm 1.21$ & $11.26 \pm 1.23$ \\
$\mathrm{CL} / \mathrm{F}(\mathrm{L} / \mathrm{h})$ & $0.86 \pm 0.21$ & $2.04 \pm 0.31$ \\
$\mathrm{~T}_{1 / 2}$ & $1.79 \pm 0.64$ & $1.13 \pm 0.47$ \\
\hline
\end{tabular}

$\mathrm{T}_{\max }(\mathrm{h})$ : time to reach maximum concentration; $\mathrm{C}_{\max }(\mathrm{ng} / \mathrm{mL})$ : maximum plasma concentration; AUC: the area under the concentration time curve; $(0-\infty)$ : from time zero to infinity; (0-24): from time zero to $24 \mathrm{~h} ; \mathrm{MRT}(\mathrm{h})$ : mean residence time; $\mathrm{Vd} / \mathrm{F}(\mathrm{L})$ : apparent distribution volume; CL/F (L/h): apparent clearance;

$\mathrm{T}_{1 / 2}$ : the half-life of drug elimination during the terminal phase. $* P<0.05$ compared with related parameter of CAMD in the alone group.

As a result, the serum concentration of CAMD metabolites increased when combined with XST.

\subsection{The Molecular Simulation between the XST and CES1A.} The docking score of ginsenoside Rgl, Rd, and notoginsenoside R1 against CES1A protein from the molecular simulation were, respectively, $-6.54,-7.12$, and -8.13 . Once the ligandreceptor complex formed, it adapted to the most stable conformation. The active site of the CES1A protein revealed that several molecular interactions could be considered responsible for the observed affinity. Hydrogen bonds could be found between the protein residue GLU815, LYS866, LUE838, and the XST ligand. These results suggest that the activity of the CES1A metabolic enzyme activity may be partially inhibited by XST. The molecular simulation results were consistent with the previous CES1A mRNA expression results.

Above all, great changes have took place in both pharmacokinetic parameters and CES1A metabolic enzyme aspect (by mRNA expression and molecular protein inhibition). Possible reasons for XST-clopidogrel interaction are complex and diverse, including gastrointestinal lesions that cause changes in drug absorption, changes in transporters responsible for uptake, efflux, and elimination, and changes in the metabolic enzymes which alter the clopidogrel metabolic rate.

Clopidogrel is an inactive prodrug that requires enzymatic conversion by carboxylesterases (CESs) and cytochrome P450 (CYP) enzymes. The liver is the organ responsible for drug metabolism enzymes. Patients have reduced hepatic metabolism of clopidogrel, via the CESs and P450 enzyme group. Currently, most of herbal medicines are administered via the oral route. While previous data showed that multiple dose of clopidogrel induced accumulation of the inactive clopidogrel carboxylic acid metabolite, the accumulation phenomenon was reduced by combination with the Chinese medicine FDDP [17]. Owing to decreased CES1A activity, the elimination of the parent drug can be changed when combined with herbal medicines.

Drug transporters also have a critical role in controlling drug exposure. Transporters are proteins facilitating the passage of drugs across biological barriers encountered during drug metabolism, among which P-glycoprotein (P-gp) can expel various drugs, resulting in multidrug resistance, and is likely to play a critical role in the uptake and absorption of substrate drugs. The intestinal absorption of ginsenoside $\mathrm{Rg} 1, \mathrm{Rd}$, and notoginsenoside R1 (the main component of XST), is enhanced by the inhibition of P-gp activity. All of the above may contribute to the changes in pharmacokinetic behavior of CAMD in rats compared with when combination with XST. However, the proposed hypotheses still need further investigation and validation.

\section{Conclusion}

Sensitive UHPLC-MS/MS and RT-PCR technique were successfully used to characterize the clopidogrel and XST herbdrug interaction in rats. Clopidogrel and XST coadministration appreciably increased the Cmax, AUC, and MRT of CAMD (the active thiol metabolite) and decreased the CES1A mRNA expression. Animal studies indicated that clopidogrel and XST coadministration produced significant herbdrug interactions in pharmacokinetic and metabolic enzyme aspect. In a word, 30-day dose of coadministration altered hepatic CES1A, and this resulted in elevated serum CAMD levels. Decreased CES1A mRNA expression and elevated serum CAMD levels were due to the XST combination.

\section{Abbreviations \\ SD: \\ XST: Xuesaitong dispersible tablets \\ CES1A: Carboxylesterase $1 \mathrm{~A}$ \\ CAMD: Clopidogrel thiol metabolite derivative \\ UHPLC-MS/MS: Ultra-high-performance liquid chromatography-tandem mass spectrometry \\ MPB: The 2-bromo-3'- methoxyacetophenone.}

\section{Data Availability}

The data used to support the findings of this study are available from the corresponding author upon request. 


\section{Conflicts of Interest}

We declare that there are no conflicts of interest associated with this manuscript and there has been no financial support that could have influenced the outcome.

\section{Authors' Contributions}

Shitang Ma and Guoliang Dai equally contributed to this work.

\section{Acknowledgments}

The authors are grateful for the financial support provided by the National Natural Science Foundation of China (Grants nos. 81403114 and 81403268), the talent fund of the Education Department of Anhui Province (Grant no. gxfx2017076), the Anhui Science and Technology University (Grant no. SPWD201602), and the Public Welfare Technology Application Research Linkage Project of Anhui Province (Grant no. 1704f0704062).

\section{References}

[1] M. Verdoia, L. Barbieri, H. Suryapranata, and G. De Luca, "Switching from Clopidogrel to Prasugrel in patients undergoing PCI: A meta-analytic overview," Platelets, vol. 27, no. 2, pp. 93-104, 2016.

[2] H. D. White, D. L. Bhatt, C. M. Gibson et al., "Outcomes With Cangrelor Versus Clopidogrel on a Background of Bivalirudin," JACC: Cardiovascular Interventions, vol. 8, no. 3, pp. 424-433, 2015.

[3] G. X. He, J. Xie, H. Jiang, W. Tan, B. Xu, and J. Integr, "Effects of Qishen Yiqi Dripping Pills (芪参益气滴丸) in Reducing Myocardial Injury and Preserving Microvascular Function in Patients Undergoing Elective Percutaneous Coronary Intervention: A Pilot Randomized Study," Chinese Journal of Integrative Medicine, vol. 24, no. 3, pp. 193-199, 2018.

[4] X. Yi, J. Lin, Y. Wang et al., "Association of Cytochrome P450 Genetic Variants with Clopidogrel Resistance and Outcomes in Acute Ischemic Stroke," Journal of Atherosclerosis and Thrombosis, vol. 23, p. 1188, 2016.

[5] L. J. Zhang, Y. Q. Zhang, X. Han et al., "Association of VEGFR-2 Gene Polymorphisms With Clopidogrel Resistance in Patients With Coronary Heart Disease," American Journal of Therapeutics, vol. 23, p. e1663, 2016.

[6] X. Hou, J. Shi, and H. Sun, "Gene polymorphism of cytochrome P450 $2 \mathrm{C} 19^{*} 2$ and clopidogrel resistance reflected by platelet function assays: a meta-analysis," European Journal of Clinical Pharmacology, vol. 70, p. 1041, 2014.

[7] Y. Li and N. Wang, "Antithrombotic effects of Danggui, Honghua and potential drug interaction with clopidogrel," Journal of Ethnopharmacology, vol. 128, p. 623, 2010.

[8] A. M. Cressman, E. M. Macdonald, K. A. Fernandes et al., "A population-based study of the drug interaction between clopidogrel and angiotensin converting enzyme inhibitors," British Journal of Clinical Pharmacology, vol. 80, no. 4, pp. 662669, 2015.

[9] O. U. Ozcan, E. Tutar, B. Candemir et al., "Overcoming aspirin resistance with loading clopidogrel earlier in elective percutaneous coronary intervention," International Journal of Angiology, vol. 24, p. 19, 2015.
[10] E. Tarkiainen, M. Holmberg, A. Tornio et al., "Carboxylesterase 1 c. $428 \mathrm{G}>$ A single nucleotide variation increases the antiplatelet effects of clopidogrel by reducing its hydrolysis in humans," Clinical Pharmacology \& Therapeutics, vol. 97, no. 6, pp. 650658, 2015.

[11] X. Wang, H. J. Zhu, and J. S. Markowitz, "Carboxylesterase 1-Mediated Drug-Drug Interactions between Clopidogrel and Simvastatin," Biological and Pharmaceutical Bulletin, vol. 38, p. 292, 2015.

[12] Z. Y. Wang, M. Chen, L. L. Zhu et al., "Pharmacokinetic drug interactions with clopidogrel: updated review and risk management in combination therapy," Therapeutics and Clinical Risk Management, vol. 11, p. 449, 2015.

[13] Y. Suzaki, N. Uemura, M. Takada et al., "The effect of carboxylesterase 1 (CES1) polymorphisms on the pharmacokinetics of oseltamivir in humans," European Journal of Clinical Pharmacology, vol. 69, no. 1, pp. 21-30, 2013.

[14] Y. S. Chhonker, C. P. Pandey, H. Chandasana et al., "Simultaneous quantitation of acetylsalicylic acid and clopidogrel along with their metabolites in human plasma using liquid chromatography tandem mass spectrometry," Biomedical Chromatography, vol. 30, no. 3, pp. 466-473, 2016.

[15] N. F. Ford, "The Metabolism of Clopidogrel: CYP2C19 Is a Minor Pathway," The Journal of Clinical Pharmacology, vol. 56, p. 1474,2016

[16] B. L. Hoh, Y. Gong, C. W. McDonough et al., "CYP2C19 and CES1 polymorphisms and efficacy of clopidogrel and aspirin dual antiplatelet therapy in patients with symptomatic intracranial atherosclerotic disease," Journal of Neurosurgery, vol. 124, no. 6, pp. 1746-1751, 2016.

[17] D.-Z. Li, Y. Zhou, and Y.-N. Yang, "Acupuncture for essential hypertension: a meta-analysis of randomized sham-controlled clinical trials," Evidence-Based Complementary and Alternative Medicine, vol. 2014, Article ID 279478, 7 pages, 2014.

[18] S. T. Ma, G. L. Dai, X. L. Bi et al., "Computational Pharmacological Study on Clopidogrel Metabolism Enzymes Influenced by Fufang Danshen Dripping Pill," Zhong Yao Cai, vol. 38, p. 1009, 2015.

[19] S. T. Ma, G. L. Dai, B. T. Sun et al., "Effect of Clopidogrel on Pharmacokinetic of Fufang Danshen Dripping Pill (FDDP)," Zhong Yao Cai, vol. 37, p. 2240, 2014.

[20] J. Y. Han, Q. Li, Z. Z. Ma, and J. Y. Fan, "Effects and mechanisms of compound Chinese medicine and major ingredients on microcirculatory dysfunction and organ injury induced by ischemia/reperfusion," Pharmacology \& Therapeutics, vol. 177, p. 146, 2017.

[21] L. J. Gan, C. H. Zhang, and M. Zhang, "Effect of intracoronary injection with xuesaitong in treating post-PCI slow-reflow phenomenon in patients with ST-segment elevation myocardial infarction," Zhongguo Zhong Xi Yi Jie He Za Zhi, vol. 30, p. 348, 2010.

[22] W. H. Frishman, J. G. Grattan, and R. Mamtani, "Alternative and complementary medical approaches in the prevention and treatment of cardiovascular disease," Current Problems in Cardiology, vol. 30, p. 383, 2005.

[23] M. M. Wang, M. Xue, Y. G. Xu et al., "Panax notoginseng saponin is superior to aspirin in inhibiting platelet adhesion to injured endothelial cells through COX pathway in vitro," Thrombosis Research, vol. 141, pp. 146-152, 2016.

[24] J.-B. Wan, S. M.-Y. Lee, J.-D. Wang et al., "Panax notoginseng reduces atherosclerotic lesions in ApoE-deficient mice and 
inhibits TNF- $\alpha$-induced endothelial adhesion molecule expression and monocyte adhesion," Journal of Agricultural and Food Chemistry, vol. 57, no. 15, pp. 6692-6697, 2009.

[25] L. Liu, L. Zhu, Y. Zou et al., "Panax notoginseng saponins promotes stroke recovery by influencing expression of Nogo$\mathrm{A}, \mathrm{NgR}$ and p75NGF, in vitro and in vivo," Biological and Pharmaceutical Bulletin, vol. 37, p. 560, 2014.

[26] T. Kabeya, W. Matsumura, T. Iwao, M. Hosokawa, and T. Matsunaga, "Functional analysis of carboxylesterase in human induced pluripotent stem cell-derived enterocytes," Biochemical and Biophysical Research Communications, vol. 486, p. 143, 2017.

[27] M. Karaźniewicz-Łada, D. Danielak, A. Tezyk, C. zaba, G. Tuffal, and F. Główka, "HPLC-MS/MS method for the simultaneous determination of clopidogrel, its carboxylic acid metabolite and derivatized isomers of thiol metabolite in clinical samples," Journal of Chromatography B, vol. 911, pp. 105-112, 2012.

[28] C. J. Peer, S. D. Spencer, D. A. VanDenBerg et al., "A sensitive and rapid ultra HPLC-MS/MS method for the simultaneous detection of clopidogrel and its derivatized active thiol metabolite in human plasma," Journal of Chromatography B, vol. 880, pp. 132139, 2012.

[29] J. B. Park, S. H. Bae, S. M. Jang et al., "Direct measurement of active thiol metabolite levels of clopidogrel in human plasma using tris(2-carboxyethyl)phosphine as a reducing agent by LCMS/MS," Journal of Separation Science, vol. 36, p. 2306, 2013.

[30] T. Maruichi, T. Fukami, M. Nakajima, and T. Yokoi, "Transcriptional regulation of human carboxylesterase $1 \mathrm{~A} 1$ by nuclear factor-erythroid 2 related factor 2 (Nrf2)," Biochemical Pharmacology, vol. 79, p. 288, 2010. 


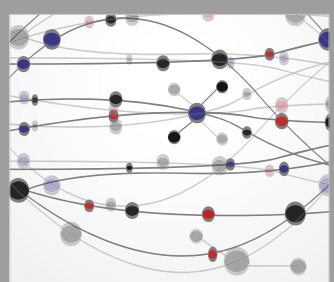

The Scientific World Journal
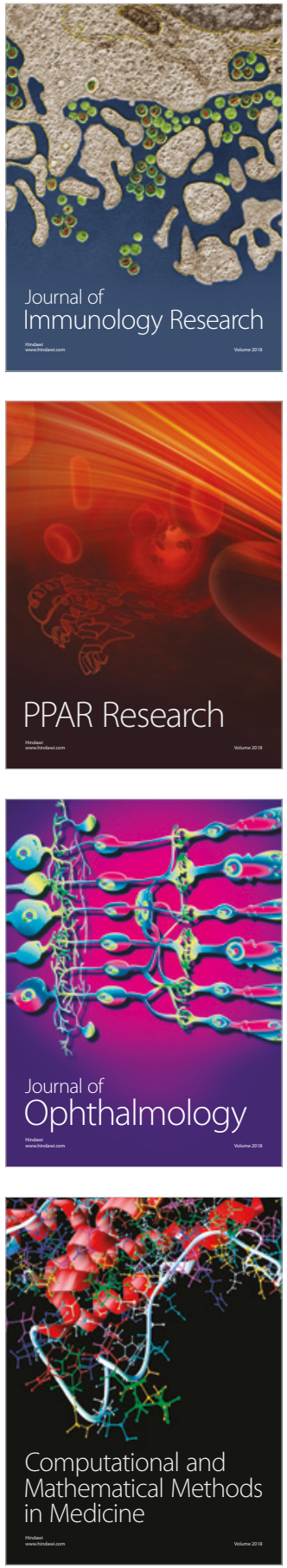

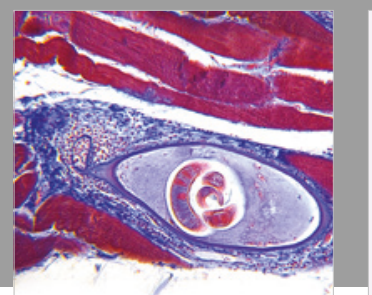

Gastroenterology Research and Practice

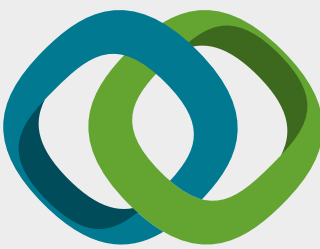

\section{Hindawi}

Submit your manuscripts at

www.hindawi.com
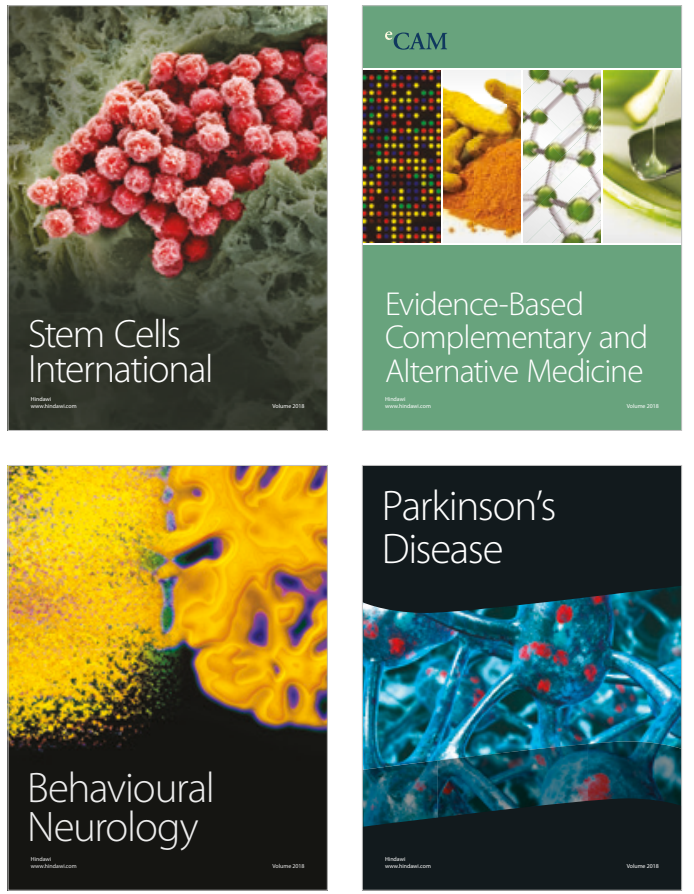

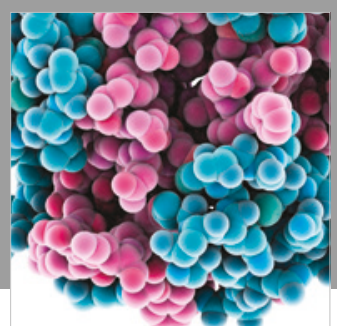

ournal of

Diabetes Research

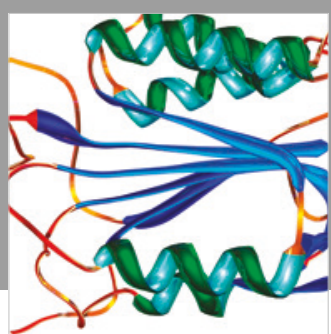

Disease Markers
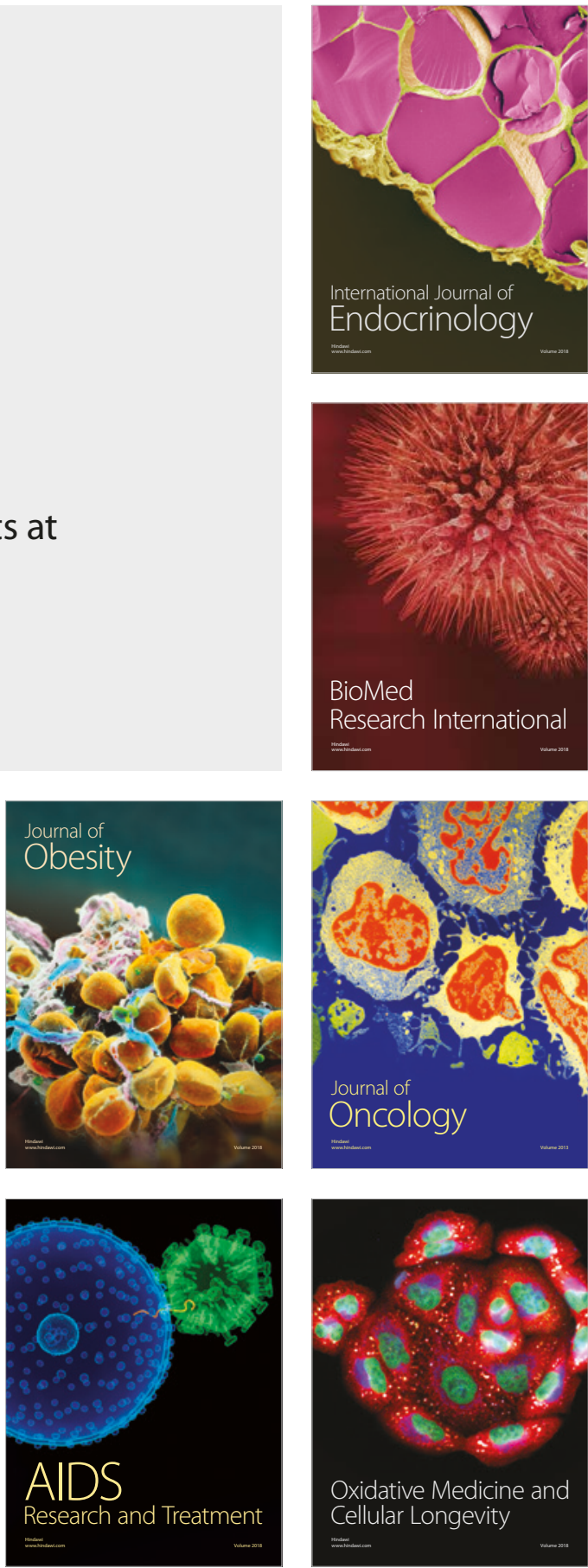